Pontifícia Universidade $_{\text {Do Rio de Janelro }}$

Mariana Maia Simoni

\title{
ACENTOS PERFORMATIVOS NA EXPERIÊNCIA TEATRAL CONTEMPORÂNEA Um diálogo construtivista com René Polesch
}

Tese de Doutorado

Tese apresentada ao Programa de Pós-Graduação em Letras da PUC-Rio como requisito parcial para obtenção do grau de Doutor em Letras.

Orientadora: Heidrun Krieger Olinto

Rio de Janeiro abril de 2011 


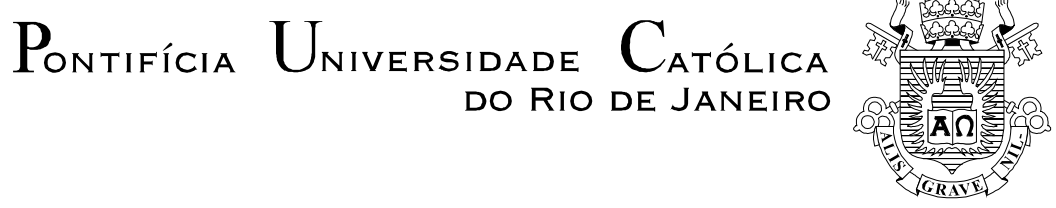

Mariana Maia Simoni

\title{
ACENTOS PERFORMATIVOS NA EXPERIÊNCIA TEATRAL CONTEMPORÂNEA Um diálogo construtivista com René Pollesch
}

\begin{abstract}
Tese apresentada como requisito parcial para obtenção do grau de Doutor pelo Programa de Pós-Graduação em Letras do Departamento de Letras do Centro de Teologia e Ciências Humanas da PUC-Rio. Aprovada pela Comissão Examinadora abaixo assinada.
\end{abstract}

Profa. Heidrun Krieger Olinto de Oliveira Orientadora

Departamento de Letras - PUC-Rio

Prof. Stephan Arnulf Baumgartel

Universidade do Estado de Santa Catarina - UDESC

Profa. Tânia Alice Feix UNIRIO

Profa. Maria Helena Vicente Werneck Cátedra Pe. António Vieira/PUC-Rio; UNIRIO

Profa. Daniela Gianna Claudia Beccaccia Versiani Departamento de Letras - PUC-Rio

Profa. Denise Berruezo Portinari Coordenadora Setorial do Centro de Teologia e Ciências Humanas - PUC-Rio

Rio de Janeiro, 11 de abril de 2011. 
Todos os direitos reservados. É proibida a reprodução total ou parcial do trabalho sem autorização da universidade, da autora e da orientadora.

\section{Mariana Maia Simoni}

Mestre em Estudos de Literatura, Bacharel e Licenciada em Letras pela Pontifícia Universidade Católica do Rio de Janeiro. Formação como atriz pelo Curso de Formação Profissional de Atores da Casa de Artes de Laranjeiras (CAL). Estágio de Doutorado com Bolsa Sanduiche da CAPES na Universitat Autónoma de Barcelona/Institut del Teatre e em Berlim. Colaboração no Grupo de Pesquisa CNPq Teorias Atuais da Literatura, inserido na Linha de Pesquisa Teorias Contemporâneas da Literatura. Participação da Câmara Setorial de Teatro (FUNARTE, 2006). Experiência docente na área de Teoria Literária. Atuação como atriz em várias peças vinculadas a formas performativas. Publicações diversas na área de estudos de literatura e artes cênicas (artigos em periódicos, anais de congresso, traduções).

Ficha Catalográfica

Simoni, Mariana Maia
Acentos performativos na
experiência teatral contemporânea: um
diálogo construtivista com René
Polesch / Mariana Maia Simoni ;
orientadora: Heidrun Krieger Olinto. -
$2011 . \quad 191$ f. : il. (color.); $30 \mathrm{~cm}$
Tese (doutorado)-Pontifícia
Universidade Católica do Rio de
Janeiro, Departamento de Letras, 2011.
Inclui bibliografia
1. Letras - Teses. 2. Teatro
performativo. 3. Teatro pós-dramático.
4. Teorias construtivistas. 5. Polesch,
René. I. Olinto, Heidrun Krieger. II.
Pontifícia Universidade Católica do Rio
de Janeiro. Departamento de Letras. III.
Título.

CDD: 800 


\section{Agradecimentos}

A Massê Llordén, por sua presença laranja na minha vida. Sempre

A Roberta Vinhas, pela amizade pré-histórica absolutamente preciosa

A Luciano Maia, seu afeto e sua cumplicidade

A Joana Simoni, por nossas conversas em trânsito

A Liane \& Romulo, meus pais, pelo apoio e amor infinitos

Aos queridos: Gabriella Allegri, Mauro \& Patrícia França, Amanda Boigues, Ana Helena Salgado, Luiza Teixeira, Cecília Wellisch, Zé Quental, Eva Randolph, Juan Urraco, Marianne Mauri, Martina Massera, Walter Valli, Javier Zaldivar, Quim Bosch, Luciana Gattass, Danusa Depes, Teresa Bastos, Carol Krause, Cristina, Inês Mello, Helô Vinadé, Daniela Versiani, Marília Rothier, Ole Erdmann, Carles Batlle, René Pollesch, Ilo, Fabio, Chang e Juanita.

Ao Departamento de Letras da PUC-Rio

Ao $\mathrm{CNPq}$

A CAPES

e a Frithwin Wagner-Lippok,

com quem aprendo muito mais do que imagina

por sua ajuda inesquecível, sua língua e seu coração 


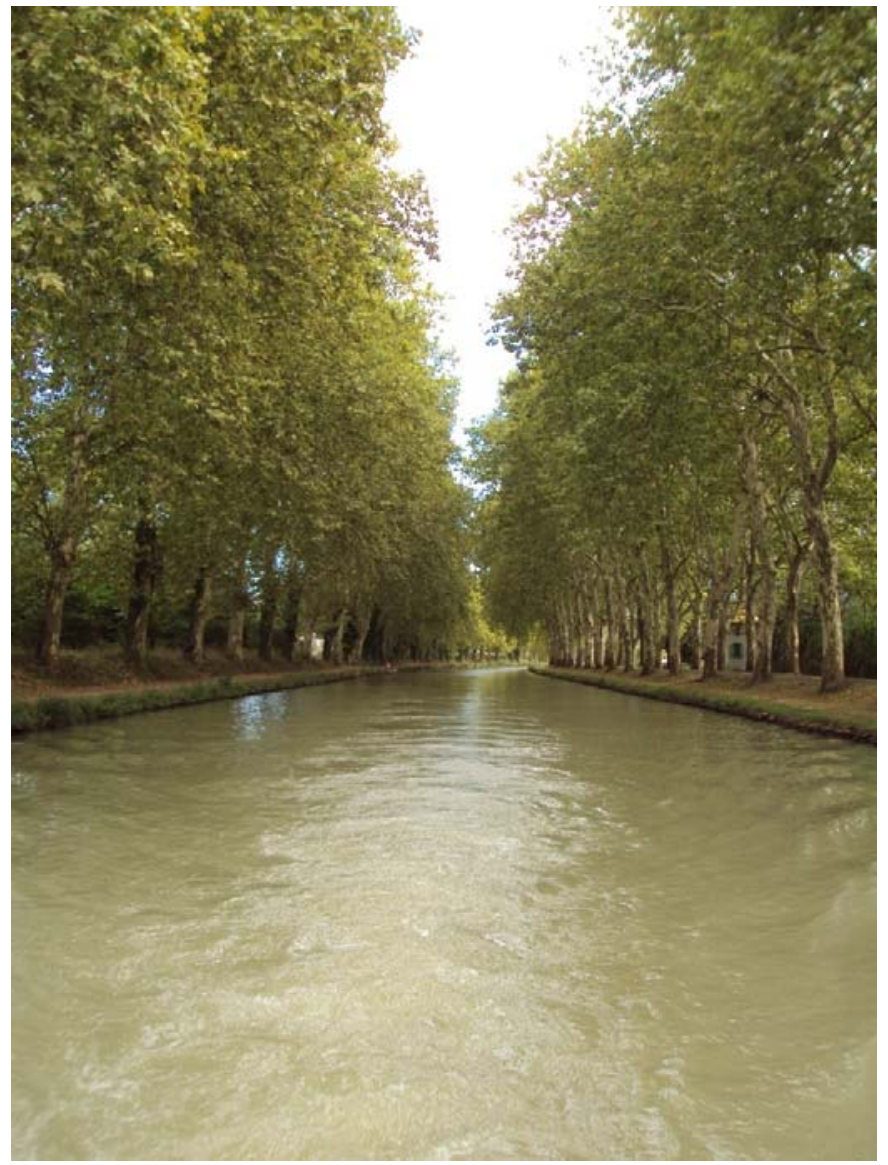

meu agradecimento para a heidrun sempre fica apertado na página a heidrun tem o olhar para o mundo mais bonito que conheço 


\section{Resumo}

Simoni, Mariana Maia. Acentos performativos na experiência teatral contemporânea. Um diálogo construtivista com René Pollesch. Rio de Janeiro, 2011. 191p. Tese de Doutorado - Departamento de Letras, Pontifícia Universidade Católica do Rio de Janeiro.

Esta tese se propõe à construção de uma perspectiva teórica que observe determinados experimentos teatrais contemporâneos a partir de sua performatividade e de possíveis deslocamentos entre a atividade teórica e a prática teatral. As proposições de Hans Ulrich Gumbrecht, bem como as pesquisas da teórica do teatro Josette Féral - buscando integrar, de forma não dicotômica, pares conceituais como razão e emoção; mente e corpo; produção de sentido e produção de presença; teoria e prática - operam como ferramentas metodológicas centrais destas investigações. A partir de uma moldura teórica sistêmica e construtivista, a tese enfoca o trabalho do diretor de teatro alemão René Pollesch, e sua inserção ambivalente na cena teatral da Alemanha do presente ao justapor repertórios teóricos complexos a elementos da cultura pop. O olhar para estas encenações procura delinear seu lugar muito particular tanto no sistema teatral quanto literário segundo alguns conceitos pertencentes a ambos os campos disciplinares, tais como "pós-dramático" (Lehmann), "performativo" (Féral) e "pós-moderno".

\section{Palavras-chave}

teatro performativo; teatro pós-dramático; teorias construtivistas; René Pollesch 


\section{Résumé}

Simoni, Mariana Maia. Dimensions performatives de l'expérience théâtrale contemporaine. Un dialogue constructiviste avec René Pollesch. Rio de Janeiro, 2011. 191p. Thèse de Doctorat - Departamento de Letras, Pontifícia Universidade Católica do Rio de Janeiro.

Cette thèse prétend aborder d'un point de vue théorique certaines expérimentations théâtrales contemporaines à partir de leur performativité et des possibles déplacements qu'elles opèrent entre l'activité théorique et la pratique théâtrale. Les contributions de Hans-Ulrich Gumbrecht, ainsi que les recherches théoriques sur le théâtre entreprises par Josette Féral - qui cherchent à intégrer, de manière non dichotomique, des doublets conceptuels comme raison et émotion; esprit et corps; production de sens et production de présence; théorie et pratique - servent d'outils méthodologiques fondamentaux dans le cadre de cette recherche. En partant des abordages proposés par les théories systémiques et constructivistes, la thèse aborde le travail du metteur en scène René Pollesch et son insertion ambigüe sur la scène théâtrale allemande contemporaine, surtout lorsqu'il met en parallèle des éléments de la culture pop et des références théoriques complexes. L'abordage de ces mises en scène cherche à délimiter un emplacement particulier tant à l'intérieur du système théâtral contemporain, comme à l'intérieur du système littéraire actuel, à partir de certains concepts appartenant aux deux champs du savoir, comme les concepts "post-dramatique" (Lehmann), "performativité" (Josette Féral) et "postmoderne".

\section{Mots clés}

théâtre performative; théâtre post-dramatique; théories constructivistes; René Pollesch 


\section{SUMÁRIO}

$\begin{array}{ll}\text { Para começar } & 11\end{array}$

$\begin{array}{lll}\text { CAPÍTULO } 1 & \text { Pressupostos teóricos } & 20\end{array}$

1.1.Primeiras nervosidades teóricas $\quad 21$

1.2.Próximas (des)esperações 44

$\begin{array}{ll}\text { 1.3.Sujando as mãos } & 57\end{array}$

CAPÍTULO 2 De etiquetas e laranjas $\quad 74$

2.1. Oscilações pós-modernas 75

2.2. Oscilações pós-dramáticas 95

$\begin{array}{ll}\text { 2.3. Oscilações performativas } & 117\end{array}$

CAPÍTULO 3 O que isso tudo tem a ver comigo? 131

3.1. Una noche de amor 132

3.2. O sotaque de Penelope Cruz 150

3.3. Centrifugações 162

$\begin{array}{ll}\text { Para (não) terminar } & 170\end{array}$

$\begin{array}{ll}\text { Referências bibliográficas } & 175\end{array}$

$\begin{array}{ll}\text { Anexo } 1 & 184\end{array}$

$\begin{array}{ll}\text { Anexo } 2 & 190\end{array}$ 
La terre est bleue comme une orange

Paul Éluard 